\title{
OPEN Single-walled carbon nanotubes as a photo-thermo-acoustic cancer theranostic agent: theory and proof of the concept experiment
}

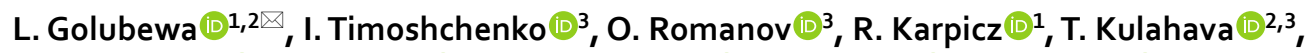 \\ D. Rutkauskas ${ }^{1}{ }^{1}$, M. Shuba $\mathbb{1}^{2,4}$, A. Dementjev ${ }^{1}$, Yu. Svirko $\mathbb{1}^{5}$ \& P. Kuzhir $\mathbb{1}^{2,5}$
}

Theranostics is the emerging field of medicine that uniquely combines diagnostic techniques and active agents to diagnose and treat medical conditions simultaneously or sequentially. Finding a theranostic agent capable to cure the affected cells and being safe for the healthy ones is the key for successful treatment. Here, we demonstrate that agglomerated single-walled carbon nanotubes (SWCNTs) are promising theranostic agent that enables photo-activated 'cold' destruction of the cancer cells keeping their environment alive. The absorption of picosecond pulses by SWCNT agglomerates results in the mechanical (due to photoacoustic effect) rather than photothermal cancer cell destruction, which was visualized by micro-Raman and ultrafast near-infrared CARS. The developed theoretical model allows us to distinguish photothermal, photoacoustic, and photothermoacoustic regimes of the cancer cell destruction, and also to optimize SWCNT-based theranostics recipe.

Lasers are widely used in biomedicine because the irradiation of living cells and tissues with coherent light beams may result in a variety of effects. Among the most examined ones are wound healing, tissue coagulation and vaporization, tissue ablation, and necrosis ${ }^{1}$. However, for the tissue embedded with micro- or nanoparticles (NPs) having the sharp absorption lines in the so-called therapeutic window of $650-1350 \mathrm{~nm}^{2}$, one can employ photothermal ${ }^{3}$, photothermoacoustic ${ }^{4}$, and photoacoustic ${ }^{5}$ effects for treatment. Owing to their ability to target tumor tissue, as well as their capacity to be manipulated by light, NPs are perfectly suitable for theranostic cancer screening, staging, and treatment ${ }^{6,7}$ using light in the near-infrared (NIR) tissue transparency window ${ }^{2}$.

Photothermal effect (or hyperthermia) is observed when light absorption in NPs results in local overheating of the surrounding tissue ${ }^{8}$ that can lead to protein denaturation, cell membrane lysis, and cell death. Although the temperature needed to destroy cancer cells in vitro is as high as $70-80^{\circ} \mathrm{C}^{9}$, even lower temperatures can be used to facilitate the release of therapeutic agents carried by $\mathrm{NPs}^{8}$. Similar to the Photothermal effect, Photothermoacoustic and Photoacoustic effects manifest themselves as the generation of acoustic waves in the NP's surrounding tissue ${ }^{10}$ due to the conversion of the absorbed light energy into heat inside a NP. In the Photothermoacoustic effect, the NIR absorption by the NP leads to the temperature increase up to thousands of degrees, the surrounding aqueous medium evaporates forming - in the nanosecond time scale-a gas bubble and leading to an acoustic perturbation and local fluid flow ${ }^{11,12}$. The Photoacoustic effect occurs when the conversion of the light energy into heat inside the NP leads to its deformation, which produces an acoustic wave in the surrounding medium, rather than forms the vapour bubble. The important advantage of this effect for cancer treatment is that in the photoacoustic regime, no heating of the surrounding medium takes place, while the cancer cell damage can be achieved by the pressure pulse generated by the overheated NP. That is search for the appropriate theranostic agent for cancer treatment implies finding NPs that enable photoacoustic regime in the NIR spectral range.

Single-walled carbon nanotubes (SWCNTs) can be such a theranostics agent because their high NIR-absorptive ability ${ }^{13}$ enables photothermal therapy ${ }^{14}$, which is much more specific and much less aggressive compared to conventional chemo- or radiotherapy. High specificity is, to a large extent, the result of SWCNTs' bioactivity

${ }^{1}$ Center for Physical Sciences and Technology, Sauletekio Ave. 3, 10257 Vilnius, Lithuania. ${ }^{2}$ Institute for Nuclear Problems, Belarusian State University, Bobruiskaya 11, 220006 Minsk, Belarus. ${ }^{3}$ Belarusian State University, Nezavisimosti Ave. 4, 220030 Minsk, Belarus. ${ }^{4}$ Tomsk State University, Lenin Ave. 36, Tomsk, Russia 634050. ${ }^{5}$ Institute of Photonics, University of Eastern Finland, Yliopistokatu 7, 80100 Joensuu, Finland. ${ }^{\bowtie}$ email: lena.golubewa@ftmc.It 
and cellular uptake $e^{15,16}$, which in turn may make them toxic ${ }^{17}$. The latter-along with the lack of solubility in aqueous media ${ }^{18}$ as well as biodistribution and pharmacokinetic issues-significantly suppresses the interest of using SWCNTs in biomedicine. However, recent advances in nanotechnology have enabled the mass production of high-quality single-chirality SWCNTs ${ }^{19}$, which can be gently cut $^{20}$, purified to the almost invisible degree of metal catalyst contamination ${ }^{21}$, and functionalized ${ }^{22}$. This has reopened interest to SWCNTs as cancer theranostics agent ${ }^{23}$ because short $(100 \mathrm{~nm})^{24}$ and properly functionalized ${ }^{25} \mathrm{CNTs}$ are significantly less toxic than long and bare ones.

The properties of biological materials may be changed considerably by embedding carbon $\mathrm{NPs}^{26}$. For example, it has been shown that the addition of $1 \%$ of CNTs enhances the thermal conductivity of aqueous suspension ${ }^{27}$. By performing SWCNTs' functionalization or varying the incubation time one may localize SWCNTs either inside the cancer cell ${ }^{28}$, on the cancer cell membrane ${ }^{29}$, or in the surrounding healthy tissue, for example, when transferring through the blood-brain barrier ${ }^{28}$. SWCNT localization and distribution is a crucial factor determining the efficiency of applied therapies, as different types of the external environment, as well as sizes of agglomerates of dispersed SWCNTs, will influence SWCNT response to NIR irradiation. In the biomedical diagnostics and therapy, either suspension of individual (or bundled) SWCNTs or micron-sized SWCNT agglomerates are used. The latter is also formed as a result of cancer cell endocytosis ${ }^{4}$ in vitro, huge SWCNT clusters are formed due to administration of high doses and local accumulation of SWCNTs in mice tissue in vivo ${ }^{30}$.

The aim of this paper is to optimize the SWCNT-based photonic theranostics recipe of detection and cold photodestruction of cancer cells by:

1. developing theory and performing a numerical simulation of the interaction of the NIR light with the SWCNTs aggregated in living cells or accumulated in the intercellular medium, and revealing the conditions of the cold photodestruction regime;

2. experimental demonstration of the cold photodestruction of the glioma C6 cancer cells that have captured the aggregated SWCNTs inside and comparison with laser treatment of the cells surrounded by individual SWCNTs situated in the extracellular medium.

\section{Results}

Theory: interaction of the laser pulses with spherical SWCNT agglomerate embedded into a cancer cell. In the Supplementary Information, we present the theory of the interaction of light with the spatially inhomogeneous (turbid) medium with embedded NPs, which have a sufficiently low (to ignore the collective effects) concentration and physical properties very different from the host medium. The performed numerical simulation for the spherical SWCNT bundles and individual SWCNTs placed in the living tissue allows us to reveal the conditions when the photothermal, photothermoacoustic and photoacoustic effects occur in the tissue embedded with the SWCNTs. Figure 1a,b shows the contour map of the temperature increase and negative pressure that can be achieved at the center of the spherical SWCNT agglomerate having a radius of $R_{0}=1 \mu \mathrm{m}$ aggregated inside the glioma cell at the initial temperature of $293 \mathrm{~K}\left(20^{\circ} \mathrm{C}\right)$. The results of the numerical simulations are summarized in Fig. 1c, which shows characteristic isobar and isotherms in the laser pulse duration-intensity plane.

The yellow line in Fig. 1c presents an isotherm, which corresponds to the agglomerate heating by $\Delta T=20 \mathrm{~K}$, when the agglomerate temperature of $T=313 \mathrm{~K}\left(40^{\circ} \mathrm{C}\right)$ achieves the functionality threshold ${ }^{31}$. The brown line in Fig. 1c corresponds to the agglomerate heating by $\Delta T=60 \mathrm{~K}$, which defines the DNA melting threshold of $353 \mathrm{~K}$ $\left(80^{\circ} \mathrm{C}\right)^{32}$ that corresponds to the cell death. In the heating range of $20 \mathrm{~K}<\Delta T<60 \mathrm{~K}$, the temperature-dependent protein denaturation ${ }^{33}$ and inactivation of repair of DNA strand breaks ${ }^{31}$ take place that causes disruption of cellular functioning and may lead to the cell death. Since selective anticancer therapy implies avoiding heating of normal tissue, the heating of the agglomerates below $\Delta T=20 \mathrm{~K}$ is preferable. The dark orange line depicts isobar corresponding to the negative pressure change threshold of $0.7 \mathrm{MPa}^{34}$. Most of biological structures have a sufficiently high resistance to compression and are considerably weaker to tensile stress. If the latter in the negative phase of a pressure wave exceeds the ultimate tensile strength of the medium, there is a high probability of local fragmentation of a cell due to micro-breaks, the formation of microbubbles, and other disturbances in the homogeneity of the structure ${ }^{35-37}$. Acoustic perturbations in the medium can be observed when the pulse duration $t_{\mathrm{p}}$ is shorter than time $t_{a} \approx 100 \mathrm{ps}$ of propagation of an acoustic wave through the SWCNT bundle. Photothermal effects prevail at $t_{\mathrm{p}}>t_{\mathrm{a}}$, because of the acoustic perturbation decays during the irradiation time. Thus, for the pulses shorter than 100 ps the dark orange line separates the parameter domain into photoacoustic non-destructive imaging ${ }^{38}$, for which nanoparticles are often used as contrast agents ${ }^{39}$, and photothermoacoustic regime.

The developed model allows us to distinguish photothermal, photothermoacoustic, and photoacoustic regimes of the interaction of light pulses with SWCNT agglomerates embedded in cytoplasm. This model confirms that the intracellular accumulation of SWCNTs and their agglomeration in micro-sized particles are necessary to implement 'cold' photo-induced cancer cell destruction keeping living bare cells intact.

The performed analysis allows us to visualize the cold photoacoustic destruction area $(\Delta T<20 \mathrm{~K}$ and $|\Delta P|>0.7 \mathrm{MPa}$ ), for the SWCNT agglomerates in the living cell. In this area, the irradiation with a laser beam will not result in tissue overheating, while the light-induced tensile stress in the SWCNT agglomerate accumulated will destroy the cancer cells. To verify the simulation results we choose for our theranostic experiment laser pulses having an intensity of $2.4 \times 10^{6} \mathrm{~W} / \mathrm{cm}^{2}$ and a duration of $10 \mathrm{ps}$ (see the star in Fig. 1c).

Experiment: interaction of picosecond laser pulses with glioma cells. We performed an experimental investigation of the interaction of laser radiation with (1) bare glioma cells (as a control sample); (2) gli- 
(a)
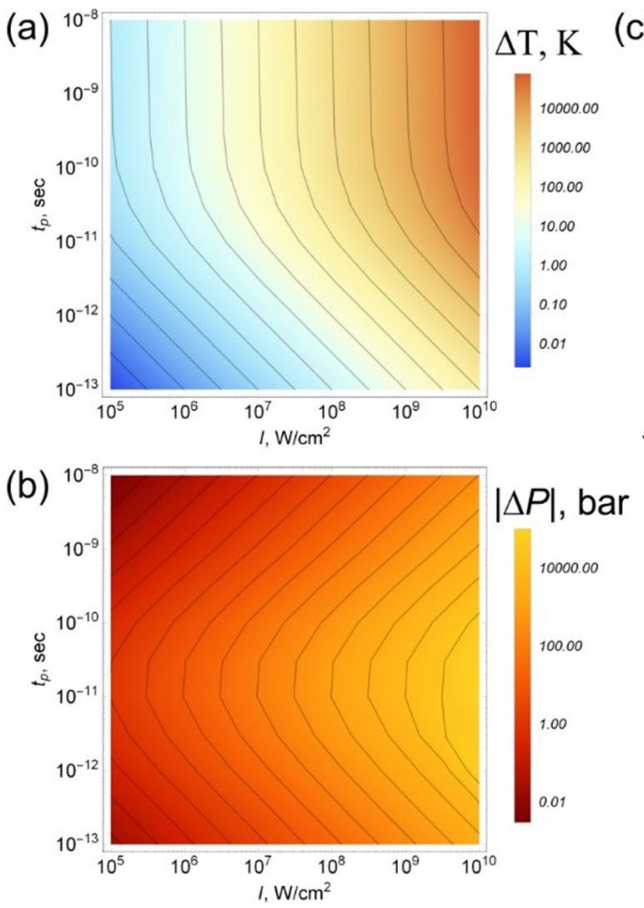

(c)

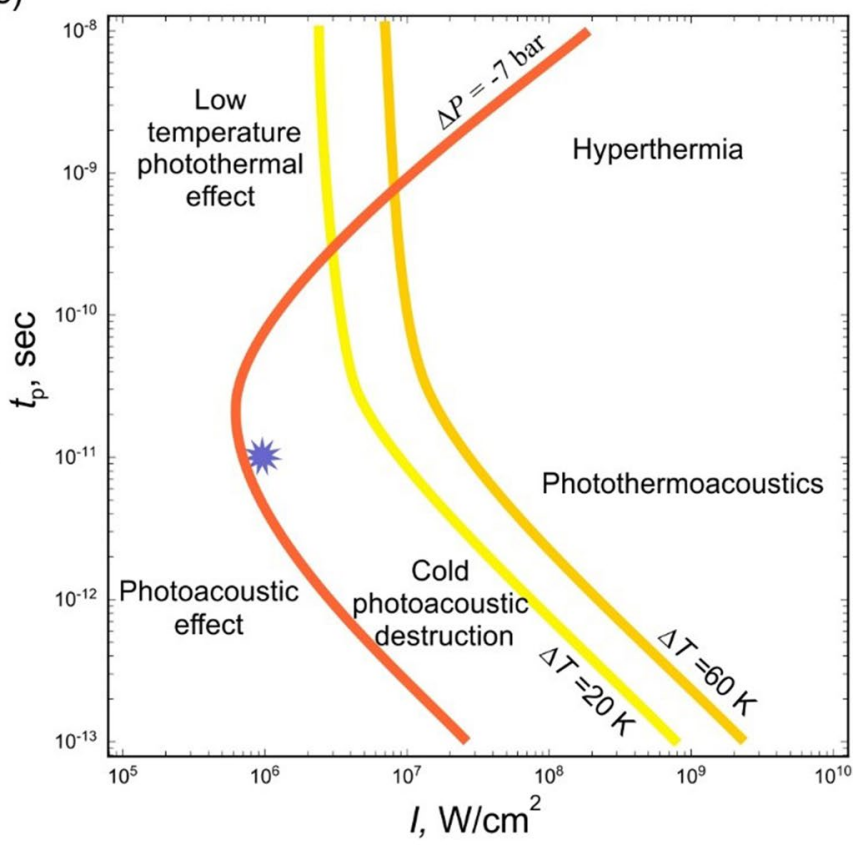

Figure 1. Numerical simulation of the interaction of laser radiation with the SWCNT agglomerate embedded into the living cell. Contour maps of the (a) temperature increase and (b) negative pressure in the SWCNT agglomerate of $R_{0}=1 \mu \mathrm{m}$ (see Supplementary Information for details) aggregated inside the glioma cell on the pulse duration/intensity plane. (c) Isotherms at $\Delta T=20 \mathrm{~K}$ and $\Delta T=60 \mathrm{~K}$, respectively, and isobar at $\Delta P=-0.7 \mathrm{MPa}$. The star corresponds to the experimental conditions.

oma cells with intracellularly accumulated and aggregated micron-sized SWCNT agglomerates; and (3) glioma cells placed into the extracellular medium with suspended individual SWCNTs.

To visualize both the distribution of the SWCNTs inside the cancer cells and their agglomeration rate we employed G-band $\left(1590 \mathrm{~cm}^{-1}\right)$ and D-band $\left(1330 \mathrm{~cm}^{-1}\right)$ of the SWCNT Raman spectrum. The G-band was used to map the SWCNT distribution in the cytoplasm of the cell. Typical images of cells after their exposure to SWCNTs for $24 \mathrm{~h}$ and a typical Raman spectrum of SWCNTs accumulated in the cells are presented in Fig. 2. It was revealed that SWCNTs were accumulated inside rat C6 glioma cells in the form of micron-sized agglomerates, SWCNTs did not enter into the nuclei and remained only in the cytoplasm.

In our experimental conditions, the excitation of the Raman signal with a continuous laser beam at $\lambda_{\text {ex }}=785 \mathrm{~nm}$ did not lead to any observable cell destruction. The laser beam was focused on the largest SWCNT agglomerate inside the cells and the cell was exposed to laser irradiation $\left(\lambda_{\text {ex }}=785 \mathrm{~nm}, 7.2 \times 10^{3} \mathrm{~W} / \mathrm{cm}^{2}\right.$, laser spot diameter $2 \mu \mathrm{m}$ ) for $10 \mathrm{~min}$. Re-scan did not show any changes in the state of the cell membrane or the distribution of agglomerates of SWCNTs inside the cell after radiation exposure, as can be seen from Fig. 2a,b. No significant change was observed in the Raman spectra of the SWCNT agglomerate used for irradiation (see Fig. 2e-1(before irradiation) and 2e-2 (after irradiation)). Increasing of the laser intensity up to $2.4 \times 10^{6} \mathrm{~W} / \mathrm{cm}^{2}$ and reduction of the scanning step from $2 \mu \mathrm{m}$ down to $0.5 \mu \mathrm{m}$ that led to an increase in the scanning time and exposure time of the cell to laser radiation, improved spatial resolution to detect the presence of smaller SWCNT agglomerates (Fig. 2c,d), which were not visualized in the Fig. 2a,b. Nevertheless, a change in the scanning parameters of the sample did not lead to the cell membrane damage and any suppression of its viability.

The same cell was exposed to laser irradiation $\left(\lambda_{\mathrm{ex}}=785 \mathrm{~nm}, 2.4 \times 10^{6} \mathrm{~W} / \mathrm{cm}^{2}\right.$, exposure time $30 \mathrm{~min}$, laser spot diameter $2 \mu \mathrm{m}$ ). An increase in intensity and exposure time did not have any effect on cell viability (Fig. $2 \mathrm{c}, \mathrm{d}$ ). Moreover, based on the analysis of the Raman spectrum of CNTs, it could be argued that there was no significant local heating of SWCNTs caused by the conversion of absorbed laser radiation into heat, as there was no increase in the D mode ${ }^{40}$ as compared to the spectrum of SWCNTs before irradiation (Fig. 2e 1-4).

Ultra-short laser pulses of high intensity can cause cell damage due to protein coagulation, vaporization, or photoablation ${ }^{41}$. Cells, cultured without SWCNTs, were exposed to picosecond laser irradiation $\left(\lambda_{\mathrm{ex}}=910.5 / 1064 \mathrm{~nm}\right)$. The same laser source was used for simultaneous fast cancer cell visualization by coherent anti-Stokes Raman spectroscopic (CARS) imaging technique (Fig. 3b) performed at $1585 \mathrm{~cm}^{-1}$ wavenumber $\left(\mathrm{C}=\mathrm{C}\right.$ bending mode in proteins $\left.{ }^{42}\right)$. To exclude the possible harmful influence of picosecond laser pulses having an intensity of $10^{6} \mathrm{~W} / \mathrm{cm}^{243}$, cell viability was determined before (Fig. 3a) and after (Fig. 3c) the irradiation using fluorescent probe propidium iodide (PI). Exposure of glioma cells to irradiation for approximately $7 \mathrm{~min}$ (time for scanning $100 \mu \mathrm{m} \times 100 \mu \mathrm{m}$ area) did not cause any plasma membrane permeabilization, as no PI fluorescence was detected (Fig. 3c).

Using CARS and fluorescent microscopy techniques we demonstrate that glioma cell plasma membrane stays intact and non-permeabilized after being irradiated with $10 \mathrm{ps}$ pulses at $\lambda_{\mathrm{ex}}=910.5 / 1064 \mathrm{~nm}$ and intensity of 

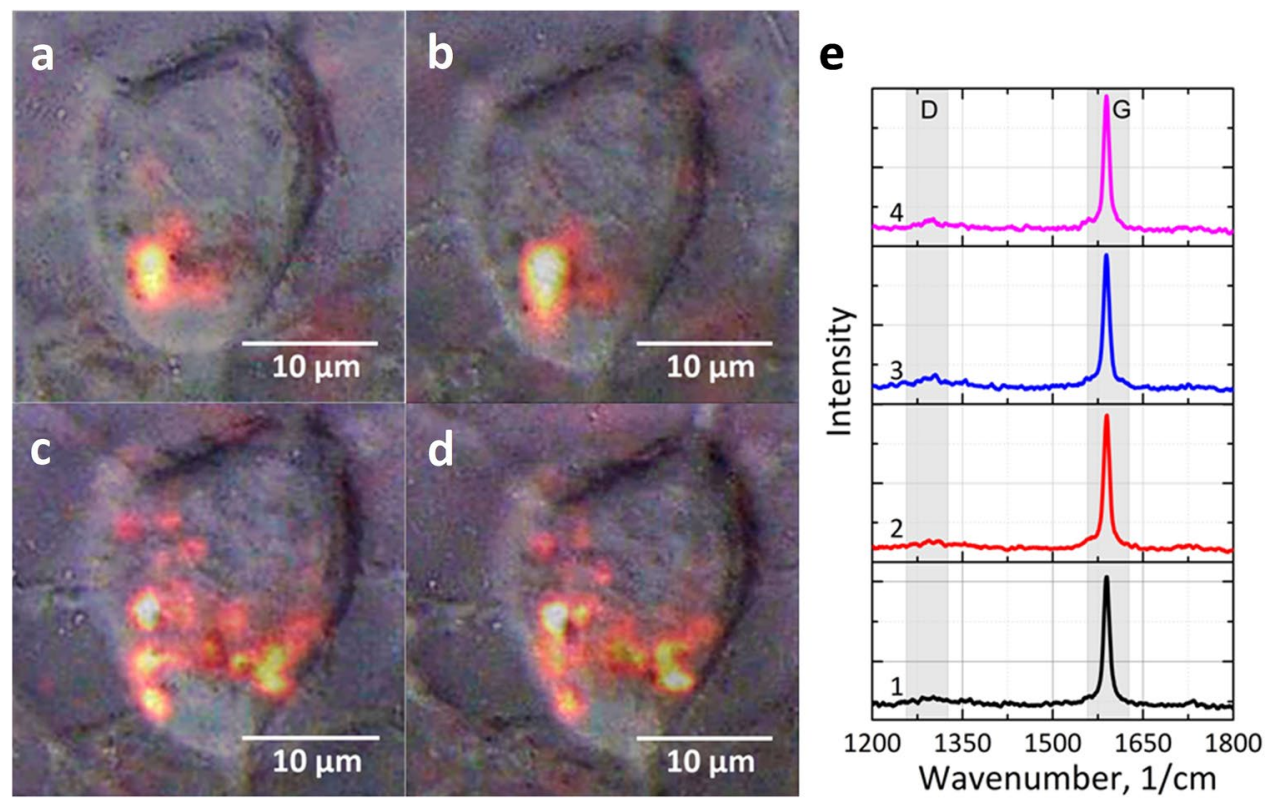

Figure 2. Intracellular accumulation of SWCNTs and structural integrity of C6 glioma cells exposed to continuous laser irradiation with $\lambda_{\mathrm{ex}}=785 \mathrm{~nm}$. (a-d) images of C6 cells in reflected light superimposed on the map of the Raman G-mode of SWCNTs; (e) Raman spectra of SWCNT agglomerates inside the cell. The cell image (a) and Raman spectrum (e-1) are taken before laser exposure. The cell image (b) and Raman spectrum $(\mathbf{e}-2)$ are taken after irradiation of the cell by a laser beam at the intensity of $7.2 \times 10^{3} \mathrm{~W} / \mathrm{cm}^{2}$ for $10 \mathrm{~min}$. The cell images (c,d) and Raman spectra (e-3,e-4) correspond to cells before and after laser irradiation for $30 \mathrm{~min}$ at the intensity of $2.4 \times 10^{6} \mathrm{~W} / \mathrm{cm}^{2}$, respectively.

$10^{6} \mathrm{~W} / \mathrm{cm}^{2}$. That is, these pulses do not have any destructive effect on the cells and can potentially be used for antitumor therapy and diagnosis.

Glioma cells cultured in the presence of SWCNTs for $24 \mathrm{~h}$ effectively accumulated SWCNTs intracellularly, and small SWCNT agglomerates were formed (see micro-Raman spectra in Fig. 2). It was shown, that exposure of cells to SWCNTs for $24 \mathrm{~h}$ and their accumulation inside the cell did not influence their viability. No PI fluorescence was detected on the cell membrane surface or somewhere in the external medium and growth surface of a Petri dish in the sample with glioma cells accumulated SWCNTs (Fig. 3d). These data indicate, that all the SWCNTs, detected by Raman microscopy (Fig. 2a-d), were located inside the cell rather than on the membrane $e^{26}$, and did not adhere to the plastic surface of the Petri dish, which could happen at the cell cultivation stage.

However, the exposition of the cells for 7 min with 10 ps laser pulses at the intensity of $10^{6} \mathrm{~W} / \mathrm{cm}^{2}$ and $1 \mathrm{MHz}$ pulse frequency $\left(\lambda_{\mathrm{ex}}=910.5 / 1064 \mathrm{~nm}\right)$ led to plasma membrane permeabilization and pore formation, detected simultaneously with irradiation by CARS imaging (Fig. 3e). The CARS signal from cells decreased, but early stages of the violation of the integrity of the cell membrane were visualized. Afterwards, using fluorescent microscopy, violations of the integrity of the cell membrane and its death were demonstrated (Fig. 3f).

To evaluate the importance of the SWCNT intracellular accumulation step, NIR pico-second pulsed light interaction with SWCNTs dispersed in the extracellular medium of cells was investigated. Cells, grown without SWCNTs, but exposed to the medium, containing $5 \mu \mathrm{g} / \mathrm{mL}$ SWCNTs, stayed viable before and after the irradiation with pico-second laser pulses for $7 \mathrm{~min}$ because the glioma cell plasma membrane was not permeabilized (Fig. $3 g-i$ ).

Therefore our experiment shows that accumulation of SWCNTs inside the cell cytoplasm appeared to be crucial in photo-induced cancer cell destruction by pico-second NIR irradiation. Pulsed irradiation of cells with intracellularly accumulated SWCNTs led to the breaks in cellular membranes and cell death, detected both by CARS and fluorescent microscopy (Fig. 3e-f). Importantly, CARS enables for simultaneous irradiation and real-time visualization of cancer cells.

Simulation of pulsed irradiation interaction with SWCNTs' micron-sized agglomerates and individually dispersed nanotubes fully proved this hypothesis. It was shown that absorption of irradiation by micron-sized agglomerate leads to photoacoustic destruction of glioma cells due to the propagation of the acoustic wave in the NP environment as shown in Fig. S.1b. If the negative pressure (volumetric extension) in the wave exceeds the threshold of $\Delta P_{\text {thresh }} \approx-0.7 \mathrm{MPa}$, destruction of the medium continuity may occur and lead to the destruction of the membrane integrity of the hosting cell. The developed model shows that laser pulses used in our experiment are capable of achieving the medium continuity destruction threshold. At the same time, local heating in the NP proximity is several degrees (see Supplementary Figure S.1a), which suggests that the photoacoustic mechanism of biostructure damage in this situation should prevail.

The simulation of the interaction of individual SWCNTs with laser pulses also fully supports our experimental results (Fig. 3g-i for experiment and Supplementary Figure S.2 for simulation). The amplitude of the generated 


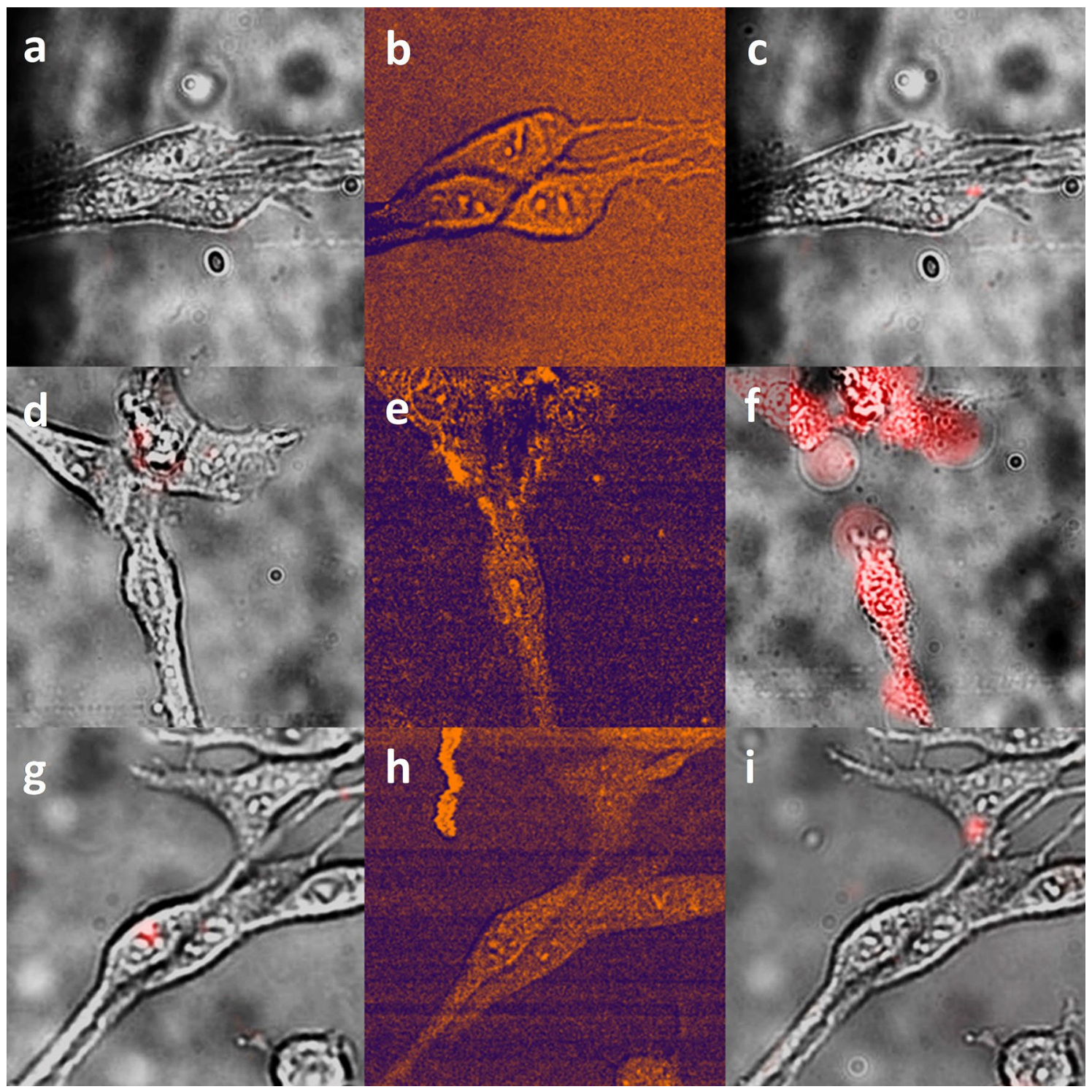

Figure 3. Photo-induced SWCNT-mediated destruction of glioma cells by NIR pico-second pulsed irradiation. (a-c) Bare C6 glioma cells; (d-f) C6 glioma cells with accumulated micron-sized SWCNT agglomerates, $(\mathbf{g}-\mathbf{i})$ C6 glioma cells in the presence of the SWCNTs suspension in the extracellular medium. (a,d,g) bright-field images superimposed with PI fluorescence images before irradiation; (b,e,h) CARS images; (c,f,i) bright-field images superimposed with PI fluorescence images after irradiation with 10 ps laser pulses $(910.5 / 1064 \mathrm{~nm}$, $10^{6} \mathrm{~W} / \mathrm{cm}^{2}$ ) for $7 \mathrm{~min}$.

pressure wave is six orders of magnitude lower than that generated in the SWCNT agglomerate. This finding allows us to conclude that in our experimental conditions, the probability of damage to the biostructures due to the generation of acoustic waves by solitary carbon nanotubes is negligible.

\section{Conclusions}

We demonstrate that glioma cells are capable of efficient accumulation of SWCNTs to relatively large agglomerates, mainly inside the formed phagolysosomes. These agglomerates enable photo-induced destruction of cancer cells using picosecond laser irradiation. It is worth noting that neither bare cancer cell nor cells with SWCNTs dispersed extracellularly are not damaged under the same irradiation conditions. We also demonstrate that CARS can be effectively used for both visualization of the cancer cell and its photo-induced SWCNT-conditioned destruction, providing strong evidence that this method is a powerful tool for nanotheranostics. The advantage of using stable SWCNT suspensions instead of conventional NPs for photoacoustic therapy is the following: in the case of NPs, not only the cell accumulated NPs will be destroyed, but also normal tissues containing these NPs associated with tumor cells will be damaged. In the case of stable SWCNT suspensions, only cells that have accumulated SWCNTs as agglomerates are destroyed. Individual nanotubes in the extracellular environment are photoacoustically inactive. Therefore, there is no damaging effect on adjacent fluids and tissues. 
The performed numerical simulation of the interaction of intense laser pulses with SWCNTs-enriched tissue may be employed to establish a common ground for the comparative study of the scattered literature data, to open a way toward increasing the efficiency of photo-induced CNT-conditioned destruction of tumors, and to reduce the cytotoxic load on the body during anticancer therapy.

\section{Methods}

SWCNT-DNA complexes. Single-walled CNTs produced by the HiPCO method (Nanointegris Technology Inc., Batch PO568) in bundles, purified from metal impurities (>98\%) were used throughout all the experiments. SWCNTs diameter varied from $0.8 \mathrm{~nm}$ to $1.4 \mathrm{~nm}$ (average $1.0 \mathrm{~nm}$ ). Metallic to semiconducting nanotubes ratio was 1:2. SWCNTs were shortened down to $100-500 \mathrm{~nm}$ in length as described $\mathrm{in}^{20}$ and functionalized by salmon DNA (Sigma-Aldrich, USA). To obtain a DNA solution, $2 \mathrm{mg}$ of the salmon DNA was placed in $6 \mathrm{ml}$ of physiological solution for 2 days at a temperature of $4^{\circ} \mathrm{C}$. Then the solution was treated with ultrasonic disperser UZDN-2 T for $30 \mathrm{~min}$ at $25 \%$ of the maximum power. Then, SWCNT agglomerates were added to $4 \mathrm{ml}$ of the DNA solution and dispersed for $4-6 \mathrm{~h}$ at low ultrasonic intensity and room temperature. The resulting suspension was centrifuged for $10 \mathrm{~min}$ at $5000 \mathrm{~g}$. In this case, SWCNT agglomerates or SWCNTs not coated with a surfactant precipitated. The supernatant was centrifuged again to remove insufficiently dispersed material. The resulting SWCNT-DNA suspension was further used for biological applications. SWCNT-DNA (SWCNTs) concentration was determined via ultraviolet-visible spectroscopy (RV2201 spectrophotometer, ZAO SOLAR, Belarus $)^{44}$. SWCNTs in suspension were stable for up to 4 months. Before each experiment SWCNTs in suspension were additionally centrifuged to remove large agglomerates formed in the suspension.

Cell culture. C6 rat glioma cells (ATCC, CCL-107) were obtained from ATCC, LGC Standards (Ogrodowa 27/29, Kielpin, Poland) and grown in DMEM/F12 (Gibco, USA) medium supplemented with 10\% newborn calf serum (Capricorn, Germany) and $80 \mu \mathrm{g} / \mathrm{mL}$ gentamycin sulfate (BelMedPreparaty, Belarus) at $37^{\circ} \mathrm{C}, 100 \%$ humidity, and $5 \% \mathrm{CO}_{2}$. For Raman spectroscopic studies $10^{5}$ cells were seeded per each Petri dish on silicon wafers and grown according to standard protocol. When cells were attached to the silicon surface, SWCNT suspension was added at a final concentration of $5 \mu \mathrm{g} / \mathrm{mL}$. SWCNT accumulation and distribution inside the cells was investigated after 18-24 h of cells growing in the presence of SWCNTs. For fluorescent microscopy and light exposure studies, cells were seeded at low density $\left(10^{4}\right.$ cells per channel) in 6 channel slide (sticky-Slide VI 0.4, Ibidi, Martinsried, Germany) with a glass coverslip as a substrate. For all experiments we used external Hepes buffer solution of the following composition (in $\mathrm{mM}$ ): $\mathrm{NaCl}-126, \mathrm{KCl}-3, \mathrm{MgSO}_{4}-2, \mathrm{CaCl}_{2}-2, \mathrm{Hepes}-10$, and glucose-6.

Raman spectroscopy of SWCNT distribution inside the cell. For Raman measurements, cells were seeded per each Petri dish on silicon $(1 \mathrm{~cm} \times 1 \mathrm{~cm})$ to avoid strong background Raman signal from the glass. After 18 or $24 \mathrm{~h}$ incubation with SWCNTs, the cellular monolayer on the slide was washed twice with PBS. Raman spectra of the cell samples were measured using Raman confocal 3D-scanning microscope NanoFinder HighEnd (Lotis TII, Belarus-Tokyo Instruments, Japan) at the excitation wavelength of $\lambda_{\text {ex }}=785 \mathrm{~nm}$ (with a maximum power of about $75 \mathrm{~mW}$ ). Exposition time was $0.5-2 \mathrm{~s}$, the scanning step was $0.5-2.0 \mu \mathrm{m}$. Data are presented using the software packages of OriginPro 8.0 (OriginLab Corp., USA), Microsoft Excel 2007, NanoFinderViewer (Lotis TII, Belarus).

Cells viability determination. To determine cell viability, propidium iodide (PI) as a fluorescent marker of cell membrane permeability was used. PI is a membrane-impermeable weakly fluorescent agent until it binds to DNA or RNA, causing 20-30 increase in fluorescence and 30-40 nm red shift of fluorescence excitation maximum at $\lambda_{\mathrm{ex}}=535 \mathrm{~nm}$ and $15 \mathrm{~nm}$ blue shift of fluorescence emission maximum at $\lambda_{\mathrm{em}}=617 \mathrm{~nm}^{45}$. When the cell plasma membrane is not intact and thermally induced pores appear, PI intercalates between the DNA bases inside the cell and strong fluorescence is detected. We used PI at the final concentration of $10^{-6} \mathrm{M}$ in the external buffer solution throughout experiments and replaced PI-containing medium by Hepes buffer without PI washing the cells with continuous fluid flow.

SWCNTs in suspension were added to 2 channels in the 6 channel slide at final concentration $5 \mu \mathrm{g} / \mathrm{mL}$ and cells were cultivated in the presence of these complexes for $24 \mathrm{~h}$ before experiments. The other channels contained DMEM/F12 with supplements, used for routine culture procedure.

Cells were irradiated under the microscope using continuous $785 \mathrm{~nm}$ laser irradiation of variable light doses on the 3rd day after seeding. For intense pulsed light treatment, a laser diode emitting a series of picosecond pulses was used. Cell samples were divided into three groups: cells without SWCNT-DNA complexes, cells exposed to SWCNT-DNA complexes for $24 \mathrm{~h}$ and accumulated SWCNTs inside the cells, and cells grown without SWCNT-DNA complexes, but irradiated in the external medium containing a high dose of SWCNTs (see Fig. 4).

Coherent anti-Stokes Raman spectroscopy (CARS). Home-made CARS system was used. Picosecond $1 \mathrm{MHz}$ laser source (EKSPLA Ltd.) with dual-wavelength was combined with Microscope Olympus IX71 and a piezo scanning system (P-517.3CL, Physik Instrumente GmbH \& Co) and utilized for raster-scanning of the sample. The exciting light was focused on the sample with an oil-immersion objective (Olympus, Plan Apochrom., 60X, NA 1.42). For detection of CARS signal the avalanche photodiode (SPCM-AQRH-14, Perkin Elmer), connected to a multifunctional PCI board (7833R, National Instruments) was used. Fundamental wavelength $(1064 \mathrm{~nm})$ and tunable-wavelength radiation $(700-1000 \mathrm{~nm})$ were used as Stokes and Pump excitation beams, respectively. Scanning of samples was performed at $1585 \mathrm{~cm}^{-1}$ wavenumber. For this, the pump beam was tuned to $910.5 \mathrm{~nm}$ and the resulting CARS signal within the spectral window from 840 to $782 \mathrm{~nm}$ 

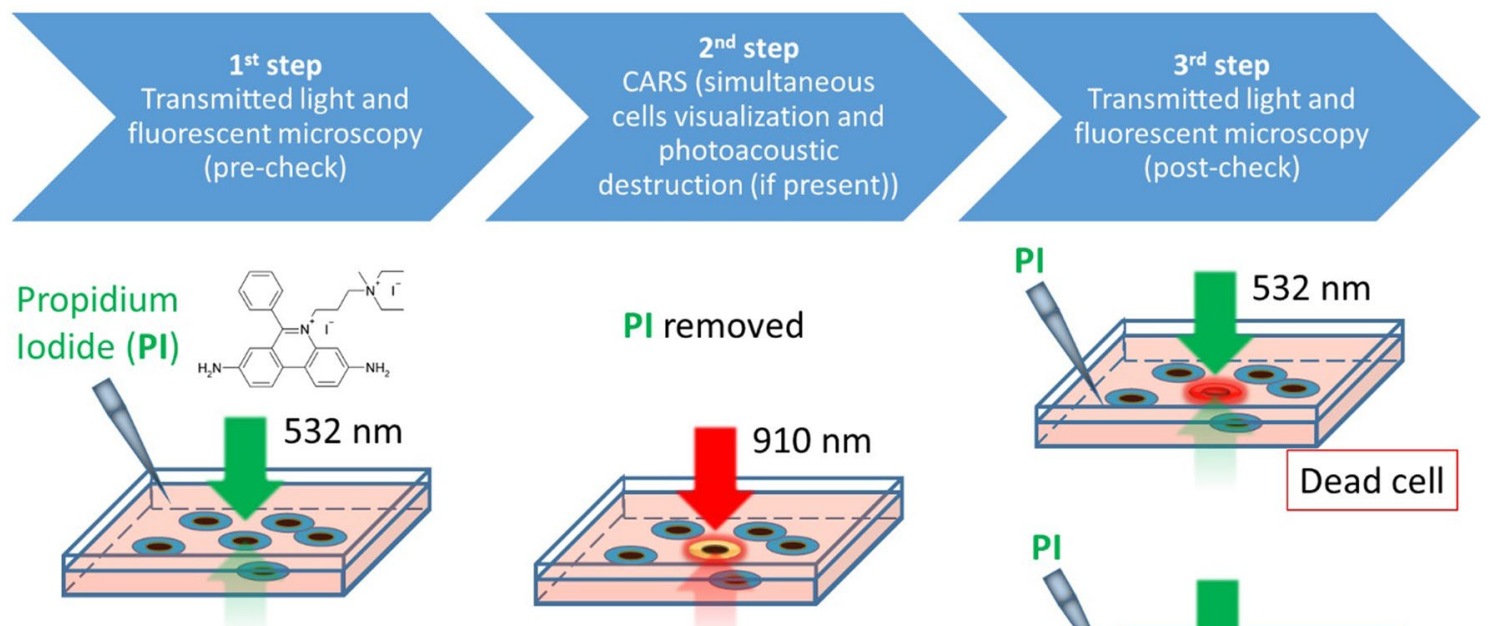

(a) “0”

(b) SWCNT-DNA (24h)

(c) SWCNT-DNA $(0 \mathrm{~h})$
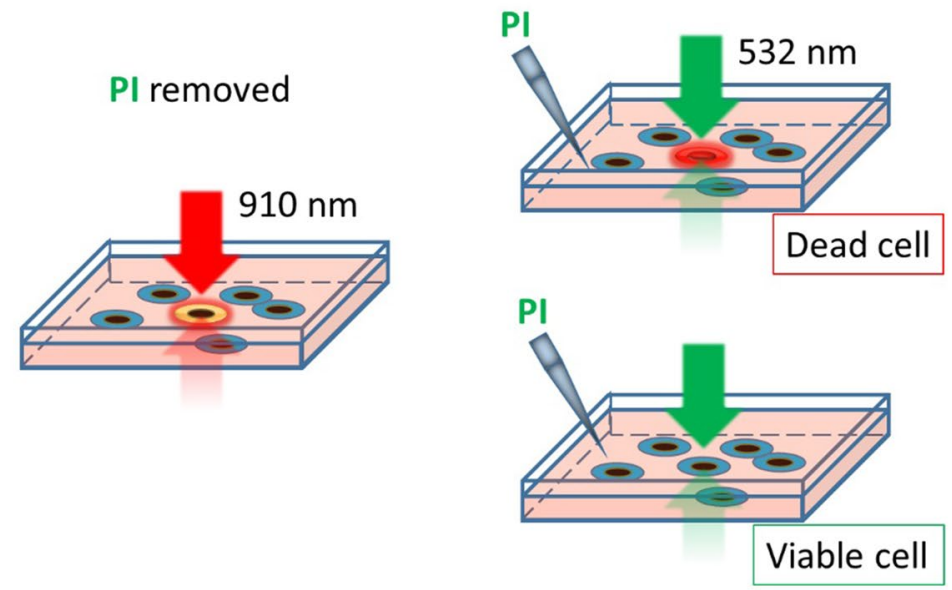

Figure 4. Experimental scheme for determination of glioma cell viability after NIR pulsed irradiation. (a) Cells without SWCNTs (control), (b) cells accumulated SWCNTs in the cytoplasm as micro-sized agglomerates, (c) cells exposed to stable suspension of individual SWCNTs.

was detected. Long-pass (cut-off at $860 \mathrm{~nm}$ ) and short-pass (cut-off at $780 \mathrm{~nm}$ ) filters were applied to spectrally separate the CARS signal. Excitation powers of $0.4 \mathrm{~mW}$ and $5 \mathrm{~mW}$ were employed for Pump and Stokes beams, respectively. Cells visualization was performed using the following set of parameters: laser-pulse duration $10 \mathrm{ps,}$ intensity $10^{6} \mathrm{~W} / \mathrm{cm}^{2}$, pulse frequency $1 \mathrm{MHz}$, scanning step $0.2 \mu \mathrm{m}$, acquisition time $1 / 500 \mathrm{~s} /$ pixel. The time for scanning the area of $100 \times 100 \mu \mathrm{m}$ using this set of parameters was $7 \mathrm{~min}$.

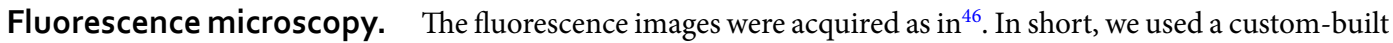
fluorescence microscope based on a commercial inverted biological Nikon Eclipse Ti-U with a $40 \times$ CFI S Plan Fluor ELWD air objective and operated in the wide-field epi-illumination mode. The $532 \mathrm{~nm}$ CW DPSS laser (Crystalaser) was used as the excitation source. The diameter of the excitation spot was about $200 \mu \mathrm{m}$. The laser power after the objective was $0.6 \mathrm{~mW}$. The excitation laser was directed into the objective by a $50 / 50$ beam splitter. The resulting fluorescence was filtered off the excitation light with the long-pass filter (HQ545LP, Chroma) and imaged with EMCCD (DU-897E-CS0-UVB, Andor). The detector integration time was $0.1 \mathrm{~s}$ and each image was an average of 20 frames.

Received: 8 October 2020; Accepted: 7 December 2020

Published online: 17 December 2020

\section{References}

1. Boulnois, J. L. Photophysical processes in recent medical laser developments: a review. Lasers Med. Sci. 1, 47-66 (1986).

2. Hemmer, E., Benayas, A., Légaré, F. \& Vetrone, F. Exploiting the biological windows: current perspectives on fluorescent bioprobes emitting above $1000 \mathrm{~nm}$. Nanoscale Horizons 1, 168-184 (2016).

3. Fong, J. F. Y., Yann, H. N. \& Sing, M. N. Carbon dots as a new class of light emitters for biomedical diagnostics and therapeutic applications. In Fullerenes, Graphenes and Nanotubes: A Pharmaceutical Approach (ed. Grumezescu, A. M.) 227-295 (Elsevier Inc., Amsterdam, 2018).

4. Khosroshahi, M. E. The effect of laser power on photothermoacoustic imaging of blood containing gold nanoparticles and deoxygenation using a frequency-domain phased array probe: an in-vitro assay. Nanosci. Technol. 2, 1-10 (2015).

5. Zhang, Y. et al. Noninvasive photoacoustic microscopy of living cells in two and three dimensions through enhancement by a metabolite dye. Angew. Chemie Int. Ed. 50, 7359-7363 (2011).

6. Frangioni, J. V. New technologies for human cancer imaging. J. Clin. Oncol. 26, 4012-4021 (2008).

7. Skripka, A. et al. Decoupling theranostics with rare earth doped nanoparticles. Adv. Funct. Mater. 29, 1807105. https://doi. org/10.1002/adfm.201807105 (2019).

8. Chen, Y. W., Su, Y. L., Hu, S. H. \& Chen, S. Y. Functionalized graphene nanocomposites for enhancing photothermal therapy in tumor treatment. Adv. Drug Deliv. Rev. 105, 190-204 (2016).

9. Eskiizmir, G., Ermertcan, A. T. \& Yapici, K. Nanomaterials: promising structures for the management of oral cancer. In Nanostructures for Oral Medicine (eds Andronescu, E. \& Grumezescu, A. M.) 511-544 (Elsevier Inc., Amsterdam, 2017).

10. Snook, R. D. \& Mitchem, L. Photoacoustic spectroscopy. In Encyclopedia of Analytical Science 2nd edn (eds Worsfold, P. et al.) 174-180 (Elsevier, Amsterdam, 2005). 
11. Holguin, S. Y., Thadhani, N. N. \& Prausnitz, M. R. Effect of laser fluence, nanoparticle concentration and total energy input per cell on photoporation of cells. Nanomed. Nanotechnol. Biol. Med. 14, 1667-1677 (2018).

12. Galisteo-González, F., Monasterio, B. G., Gil, D., Valle, M. \& Goñi, F. M. Photoacoustic effect applied on model membranes and living cells: direct observation with multiphoton excitation microscopy and long-term viability analysis. Sci. Rep. 10, 1-9 (2020).

13. Wei, X. et al. Experimental determination of excitonic band structures of single-walled carbon nanotubes using circular dichroism spectra. Nat. Commun. 7, 1-9 (2016).

14. Liang, C. et al. Tumor metastasis inhibition by imaging-guided photothermal therapy with single-walled carbon nanotubes. Adv. Mater. 26, 5646-5652 (2014).

15. Shvedova, A. A. et al. Mechanisms of pulmonary toxicity and medical applications of carbon nanotubes: Two faces of Janus?. Pharmacol. Ther. 121, 192-204 (2009).

16. Chelnokova, I. A. et al. Effect of single-walled carbon nanotubes on the structural, physical, and mechanical properties of rat glial cell surface. J. Nanopart. Res. 22, 144 (2020).

17. Eatemadi, A. et al. Carbon nanotubes: Properties, synthesis, purification, and medical applications. Nanoscale Res. Lett. 9, 1-13 (2014).

18. Wu, H. Q., Wei, X. W., Shao, M. W. \& Gu, J. S. Synthesis of zinc oxide nanorods using carbon nanotubes as templates. J. Cryst. Growth 265, 184-189 (2004)

19. Huang, J. Q., Zhang, Q., Zhao, M. Q. \& Wei, F. A review of the large-scale production of carbon nanotubes: the practice of nanoscale process engineering. Chin. Sci. Bull. 57, 157-166 (2012).

20. Shuba, M. V. et al. Soft cutting of single-wall carbon nanotubes by low temperature ultrasonication in a mixture of sulfuric and nitric acids. Nanotechnology 23, 495714 (2012).

21. Hou, P. X., Liu, C. \& Cheng, H. M. Purification of carbon nanotubes. Carbon N. Y. 46, 2003-2025 (2008).

22. Zhou, Y., Fang, Y. \& Ramasamy, R. P. Non-covalent functionalization of carbon nanotubes for electrochemical biosensor development. Sensors 19, 392 (2019).

23. Augustine, S. et al. Recent advances in carbon based nanosystems for cancer theranostics. Biomater. Sci. 5, 901-952 (2017).

24. Kostarelos, K. The long and short of carbon nanotube toxicity. Nat. Biotechnol. 26, 774-776 (2008).

25. Vardharajula, S. et al. Functionalized carbon nanotubes: biomedical applications. Int. J. Nanomed. 7, 5361-5374 (2012).

26. Gong, F. et al. Effective thermal transport properties in multiphase biological systems containing carbon nanomaterials. RSC Adv. 7, 13615-13622 (2017).

27. Ding, Y., Alias, H., Wen, D. \& Williams, R. A. Heat transfer of aqueous suspensions of carbon nanotubes (CNT nanofluids). Int. J. Heat Mass Transf. 49, 240-250 (2006).

28. Cui, X., Wan, B., Yang, Y., Ren, X. \& Guo, L. H. Length effects on the dynamic process of cellular uptake and exocytosis of singlewalled carbon nanotubes in murine macrophage cells. Sci. Rep. 7, 1518. https://doi.org/10.1038/s41598-017-01746-9 (2017).

29. Roxbury, D., Jena, P. V., Shamay, Y., Horoszko, C. P. \& Heller, D. A. Cell membrane proteins modulate the carbon nanotube optical bandgap via surface charge accumulation. ACS Nano 10, 499-506 (2016).

30. Principi, E. et al. Systemic distribution of single-walled carbon nanotubes in a novel model: Alteration of biochemical parameters, metabolic functions, liver accumulation, and inflammation in vivo. Int. J. Nanomed. 11, 4299-4316 (2016).

31. Lepock, J. R. \& Borrelli, M. J. How do cells respond to their thermal environment?. Int. J. Hyperth. 21, 681-687 (2005).

32. Marmur, J. \& Doty, P. Determination of the base composition of deoxyribonucleic acid from its thermal denaturation temperature. J. Mol. Biol. 5, 109-118 (1962).

33. Schön, A., Clarkson, B. R., Jaime, M. \& Freire, E. Temperature stability of proteins: Analysis of irreversible denaturation using isothermal calorimetry. Proteins Struct. Funct. Bioinf. 85, 2009-2016 (2017).

34. Zheltov, G. I., Romanov, O. G. \& Bourko, W. D. Physical fundamentals of low-temperature laser ablation of biotissues: quasicontinuous radiation mode. Dokl. Natl. Acad. Sci. Belarus 63, 421-429 (2019).

35. Zheltov, G. I., Lisinetskii, V. A., Grabtchikov, A. S. \& Orlovich, V. A. Low-threshold cavitation in water using IR laser pulse trains. Appl. Opt. 47, 3549-3554 (2008).

36. Sánchez, D. et al. Noncontact measurement of the local mechanical properties of living cells using pressure applied via a pipette. Biophys. J. 95, 3017-3027 (2008).

37. Wang, M. et al. Sonoporation-induced cell membrane permeabilization and cytoskeleton disassembly at varied acoustic and microbubble-cell parameters. Sci. Rep. 8, 1-12 (2018).

38. Strohm, E. M., Moore, M. J. \& Kolios, M. C. High resolution ultrasound and photoacoustic imaging of single cells. Photoacoustics 4, 36-42 (2016).

39. Li, W. \& Chen, X. Gold nanoparticles for photoacoustic imaging. Nanomedicine 10, 299-320 (2015).

40. Molina-Duarte, J., Espinosa-Vega, L. I., Rodríguez, A. G. \& Guirado-López, R. A. Raman spectra of single walled carbon nanotubes at high temperatures: pretreating samples in a nitrogen atmosphere improves their thermal stability in air. Phys. Chem. Chem. Phys. 19, 7215-7227 (2017).

41. Steiner, R. Laser-tissue interactions. In Laser and IPL Technology in Dermatology and Aesthetic Medicine (eds Raulin, C. \& Karsai, S.) 23-36 (Springer, Berlin, 2011).

42. Prats Mateu, B. et al. Label-free live cell imaging by confocal Raman microscopy identifies CHO host and producer cell lines. Biotechnol. J. 12, 1600037 (2017).

43. Schneckenburger, H. et al. Light exposure and cell viability in fluorescence microscopy. J. Microsc. 245, 311-318 (2012).

44. Golubewa, E. N., Shuba, M. V., Vasilieu, M. V. \& Kulahava, T. A. Application of Raman spectroscopy for analysis of carbon nanotube distribution in living cells. J. Appl. Spectrosc. 85, 1121-1127 (2019).

45. Crowley, L. C. et al. Dead cert: measuring cell death. Cold Spring Harb. Protoc. 1064-1072, 2016. https://doi.org/10.1101/pdb. top070318 (2016).

46. Golubewa, L. et al. Surface-enhanced Raman spectroscopy of organic molecules and living cells with gold-plated black silicon. ACS Appl. Mater. Interfaces 12, 50971-50984 (2020).

\section{Acknowledgements}

This work was financially supported by Horizon 2020 RISE DiSeTCom [Project No 823728], the Research Council of Lithuania Foundation [Joint Project No S-LB-19-4], the Belarusian Republican Foundation for Fundamental Research [Project No F18R-141], the Academy of Finland Flagship Programme, Photonics Research and Innovation (PREIN) [Project No 320166], the Academy of Finland [Project No 298298]. PK is supported by Horizon 2020 IF TURANDOT [Project No 836816]. MS is supported by Tomsk State University Competitiveness Improvement Program. We are thankful for Alesia Paddubskaya (Institute for Nuclear Problems, Belarusian State University, Minsk, Belarus) for fruitful discussions. 


\section{Author contributions}

L.G., T.K. and P.K. worked out the concept of the research. L.G., R.K., T.K., M.S., D.R. and A.D. conceived and designed the experimental study. Theoretical calculations were performed by I.T. and O.R. M.S. prepared the SWCNT-DNA complexes with the assistance of L.G. L.G. and T.K. designed and performed cell viability assay. D.R. performed the fluorescence measurement. A.D. performed the CARS measurements. L.G. and M.S. performed Raman spectroscopic measurements. The manuscript was written through the contributions of all authors. P.K. and Y.S. provided manuscript review and editing. All authors have approved the final version of the manuscript.

\section{Competing interests}

The authors declare no competing interests.

\section{Additional information}

Supplementary Information The online version contains supplementary material availlable at https://doi. org/10.1038/s41598-020-79238-6.

Correspondence and requests for materials should be addressed to L.G.

Reprints and permissions information is available at www.nature.com/reprints.

Publisher's note Springer Nature remains neutral with regard to jurisdictional claims in published maps and institutional affiliations.

(c) Open Access This article is licensed under a Creative Commons Attribution 4.0 International License, which permits use, sharing, adaptation, distribution and reproduction in any medium or format, as long as you give appropriate credit to the original author(s) and the source, provide a link to the Creative Commons licence, and indicate if changes were made. The images or other third party material in this article are included in the article's Creative Commons licence, unless indicated otherwise in a credit line to the material. If material is not included in the article's Creative Commons licence and your intended use is not permitted by statutory regulation or exceeds the permitted use, you will need to obtain permission directly from the copyright holder. To view a copy of this licence, visit http://creativecommons.org/licenses/by/4.0/.

(C) The Author(s) 2020 